\title{
Variación genética de lipoproteínas y lípidos plasmáticos
}

\author{
José Luis Santos \\ Departamento de Nutrición, Diabetes y Metabolismo \\ Facultad de Medicina Pontificia Universidad Católica de Chile.
}

Recibido el 26 de Enero de 2010, Aceptado el 10 de marzo de 2010

Rev Chil Cardiol 2010; 29: 88-93

A pesar de las importantes influencias que ejercen la dieta, los estilos de vida y los factores ambientales en las dislipidemias de origen multifactorial, existen también numerosas evidencias que apoyan la participación de factores genéticos en la variabilidad de los lípidos plasmáticos ${ }^{1}$. Los estudios de pares de gemelos monocigóticos $(\mathrm{MZ})$ y dicigóticos (DZ) indican en términos generales que existe una mayor similitud en las concentraciones de lípidos plasmáticos en gemelos $\mathrm{MZ}$ en comparación con los gemelos $\mathrm{DZ}$, lo que indicaría que estos rasgos se encuentran parcialmente bajo control genético ${ }^{2-4}$.

Es importante considerar la variabilidad de los lípidos plasmáticos en la salud de las personas, dado que el riesgo de desarrollar Enfermedad Cardiovascular (ECV) se relaciona de forma directa con los niveles de colesterol total y colesterol-LDL, e inversamente con los niveles de colesterol-HDL ${ }^{5}$. Aunque los efectos del colesterol-LDL y HDL sobre el riesgo cardiovascular están ampliamente documentados, el efecto causal de las variaciones del colesterol-HDL se encuentra aún en debate dado que algunos desórdenes del metabolismo de lípidos caracterizados por niveles muy bajos de esta fracción lipídica (por ejemplo, en las deficiencias genéticas de ABCA1, LCAT y la mutación Milano del gen APOA1) no parecen presentar un elevado riesgo de ECV6-8. Por otro lado, los niveles elevados de triglicéridos (TG), tanto basales como postprandiales, parecen estar asociados de forma independiente con el riesgo de ECV, aunque con un efecto de menor magnitud que el del colesterol-LDL ${ }^{9}$. En este sentido, algunos autores han sugerido que los valores de TG postprandiales podrían tener un efecto de mayor relevancia que los TG en ayunas como predictores del riesgo de $\mathrm{ECV}^{10}$, existiendo adicionalmente un control genético en la modulación de la lipemia postprandial ${ }^{11}$.

Valores de trigliceridemia excesivamente altos son propios de cinco de los seis clásicos fenotipos de las llamadas hiperlipoproteinemias de Frederickson (tipos 1, 2B, 3, 4 y 5), siendo la tipo 2 (hipercolesterolemia familiar) la única hiperlipoproteinemia que no presenta hipertrigliceridemia de forma característica ${ }^{12}$. El ejemplo más clásico de influencia genética sobre los niveles de TG se observa en la hiperlipoproteinemia tipo 1, que se produce por una alteración en la actividad de la Lipoproteína Lipasa $(\mathrm{LPL})^{13}$. Esta enzima juega un papel fundamental en la regulación de lípidos plasmáticos, ya que es la encargada de la hidrólisis de los TG empaquetados en los quilomicrones y en las partículas VLDL para producir ácidos grasos que serán captados por el músculo y el tejido $\operatorname{adiposo}^{14}$.

Los pacientes afectados con hiperlipoproteinemia tipo 1 pueden presentar pancreatitis aguda, estando

Correspondencia: Prof. José Luis Santos

Departamento de Nutrición, Diabetes y Metabolismo.

Facultad de Medicina. Pontifica Universidad Católica de Chile

Correo Electrónico: jsantos@med.puc.cl 
aún en discusión cuál es el impacto real de esta enfermedad sobre el riesgo de ECV. Un fenotipo similar ocurre en la deficiencia familiar del gen APOC2, que es un activador de la LPL en condiciones fisiológicas. Se han descrito defectos genéticos raros en APOC2 que, en estado autosómico recesivo, generan altos niveles de TG en plasma similares a los encontrados en la deficiencia de $\mathrm{LPL}^{15}$. En la hiperlipoproteinemia 3 (también llamada disbetalipoproteinemia), existe típicamente un estado homocigoto para el alelo E2 del gen APOE, que se acompaña de otros factores precipitantes de dislipidemias que pueden incluir tanto factores ambientales, concurrencia de otras patologías (obesidad, diabetes), así como adicionales polimorfismos genéticos de riesgo tales como las variantes $-1131 \mathrm{~T}>\mathrm{C}$ y $\mathrm{S} 19 \mathrm{~W}$ del gen APOA5 ${ }^{1}$. En el extremo opuesto de la distribución poblacional de TG, también se han descrito casos clínicos con muy bajos niveles de triglicéridos plasmáticos, tales como los que se presentan en la abetalipoproteinemia, la hipobetalipoproteinemia y la enfermedad de retención de quilomicrones, producidas respectivamente por mutaciones en MTTP (proteína encargada del ensamblaje de los quilomicrones en el intestino), mutaciones en el gen APOB (componente característico de los quilomicrones en su isoforma editada B48) o mutaciones en el gen SAR1B' ${ }^{1}$. En un estudio de barrido de genoma completo en una población aislada, se ha descrito recientemente una mutación en el gen humano APOC3, que codifica para una proteína que actúa como inhibidora de la $\mathrm{LPL}^{17,18}$. Este estudio pudo establecer una asociación entre valores muy bajos de TG postprandiales con la presencia de una variante genética cercana al agrupamiento de genes APOA1-C3-A4-A5 del cromosoma 11, en el que finalmente se identificó una mutación en estado heterocigoto en APOC3 relacionado con una aparente protección cardiovascular ${ }^{18}$. Otras mutaciones de baja frecuencia relacionadas con alteraciones en los niveles de TG se han encontrado en los genes LMF1, GPIHBP1,
ANGPTL3 y ANGPTL41. Es probable que existan otros genes desconocidos hasta el momento que puedan cargar con mutaciones responsables de cambios drásticos en la concentración de TG plasmáticos.

Se han descrito estrategias para la búsqueda de nuevos genes y mutaciones responsables de enfermedades, como el enfoque de mapeo por homocigosidad para enfermedades de herencia recesiva en familias consanguíneas ${ }^{19}$ o la reciente posibilidad de aplicar nuevas técnicas de secuenciación masiva que han permitido la determinación de la secuencia completa del exoma (total de las secuencias codificantes en el genoma humano ${ }^{20}$. Esta última estrategia ya ha mostrado su utilidad al identificar el gen y la mutación res-ponsables de una enfermedad mendeliana cuya etiología genética era desconocida hasta el momento ${ }^{20}$.

La investigación en epidemiología genética de enfermedades y rasgos multifactoriales en los últimos 20 años, ha estado dominada por los estudios de ligamiento de tipo no-paramétrico que consisten en barridos genómicos de marcadores de tipo microsatélite en familias, así como por los estudios de casos-controles focalizados en la variabilidad genética de genes candidatos ${ }^{21,22}$. Mientras que los estudios de ligamiento han sido muy exitosos en la identificación de genes involucrados en enfermedades mendelianas, su efectividad ha sido muy limitada en el estudio de rasgos multifactoriales ${ }^{23}$.

Por otro lado, los estudios de casos y controles en genes candidatos han sido criticados por limitaciones metodológicas que se han traducido en un número elevado de falsos positivos y en una baja replicabilidad ${ }^{24}$.

En los últimos años, se ha materializado la factibilidad técnica de realizar estudios de asociación en sujetos sin relación familiar en los que se realiza un barrido del genoma completo con marcadores 
genéticos de variaciones simples de tipo SNP (acrónimo de Single Nucleotide Polymorphism)25. Actualmente existen diferentes paneles de cobertura del genoma que permiten la determinación de un número de SNPs superior al millón de genotipos por muestra (http://www.illumina.com y http://www.affymetrix.com).

El objetivo de los estudios de asociación de genoma completo es encontrar marcadores genéticos que se asocien a rasgos o enfermedades, a través del fenómeno de asociación alélica (también conocido como desequilibrio de ligamiento) existente entre los SNPs determinados en el ensayo y las variantes genéticas causales de la enfermedad ${ }^{25}$.

La racionalidad de los estudios de asociación de barrido de genoma completo se basa en la hipótesis "enfermedad común-genotipo común", que afirma que existen diferentes variantes genéticas comunes que contribuirían a incrementar el riesgo de enfermar con efectos individuales de baja magnitud ${ }^{22}$. En relación a los estudios de asociación de genoma completo con TG plasmáticos, se han encontrado las señales más significativas en los genes LPL, el agrupamiento APOA1-C3-A4-A5 del cromosoma 11, y los genes APOB, GCKR, ANGPTL3 y MLXIPL, así como en otros genes con efecto de menor magnitud ${ }^{26-29}$.

Aunque la aplicación de barridos genómicos en los estudios de asociación de genoma completo ha significado un importante avance en la genética de las dislipidemias, también es importante señalar que estos avances probablemente van a impulsar nuevos estudios epidemiológicos en genes candidatos focalizados en la estimación correcta de la magnitud del riesgo de enfermar (riesgo relativo, odds ratio).

Los meta-análisis ${ }^{30}$ y los estudios centrados en el genotipado intensivo de genes previamente involucrados en la enfermedad cardiovascular ("gene centric" HumanCVD BeadChip de Illumina, con cerca de 50000 SNPs en aproximadamente
2100 genes) han puesto de manifiesto la relevancia de la variación genética de los genes candidatos conocidos relacionados con la ECV, confirmando las asociaciones ya descritas en relación a los TG plasmáticos (descritas más arriba), así como las asociaciones ya conocidas entre APOEAPOC1-APOC4-APOC2 (cromosoma 19), LDLR, APOB y PCSK9 con colesterol-LDL (y niveles de apolipoproteína B), así como las asociaciones entre CEPT y LPL con los niveles de colesterol-HDL (y niveles de apolipoproteína $\mathrm{Al})^{1,31}$. Adicionalmente, la re-secuenciación de estos genes en familias con dislipidemias severas puede ser útil en la búsqueda de mutaciones responsables de estas patologías. Se ha sugerido que la variabilidad de los TG plasmáticos está determinada por mutaciones de muy baja frecuencia con un efecto de gran magnitud, junto con otras variantes genéticas de tipo SNP con un efecto de menor magnitud y con una mayor frecuencia poblacional ${ }^{1}$. Además de la variabilidad genética de sustituciones simples de tipo SNP, también es preciso considerar otro tipo de variación de gran relevancia que consiste en el número de copias variables de segmentos cromosómicos amplios (mayores de $1 \mathrm{~Kb}$ ) llamados en la literatura "Copy-Number Variations" (CNV). Los CNV tienen un enorme potencial en la explicación de la arquitectura genética de muchos rasgos y enfermedades dado que podrían afectar al equilibrio de dosis alélica, a la disrupción de genes y a la expresión génica ${ }^{32}$. En el gen LPA que codifica para la lipoproteína $L p(a)$, se ha descrito un $C N V$ (y un conjunto de SNPs asociados), cuya variabilidad en el número de copias se encuentra asociada a los niveles de $L p(a)$, al tamaño de esta lipoproteína, y al riesgo cardiovascular ${ }^{33,34}$. Como recapitulación a lo anteriormente expuesto, la Figura 1 muestra las diferentes opciones de diseño de estudio y tipos de marcadores genéticos que han sido usados en el análisis de la etiología genética de la dislipidemia humana. 
Figura 1.- DISEÑOS DE ESTUDIO Y USO DE MARCADORES GENÉTICOS EN EL ESTUDIO DE LA ETIOLOGÍA GENÉTICA DE LAS DISLIPIDEMIAS HUMANAS

\begin{tabular}{|c|c|}
\hline ESTUDIOS GLOBALES DEL GENOMA & ESTUDIOS DE GENES CANDIDATOS \\
\hline Dislipidemias monogénicas & Dislipidemias monogénicas y multifactoriales \\
\hline $\begin{array}{l}\text { Barridos genómicos en familias: mapeo por } \\
\text { homocigosidad y ligamiento paramétrico } \\
\text { (cita 19) }\end{array}$ & $\begin{array}{l}\text { Re-secuenciación de genes candidatos } \\
\text { (rvisados en cita 1) }\end{array}$ \\
\hline $\begin{array}{l}\text { Secuenciación completa del exoma } \\
\text { (cita 20) }\end{array}$ & $\begin{array}{l}\text { Estudios epidemiológicos clásicos (caso- } \\
\text { control, cohorte) } \\
\text { SNP (Cita 35): CNV (cita 33) }\end{array}$ \\
\hline \multicolumn{2}{|l|}{ Dislipidemias multifactoriales } \\
\hline $\begin{array}{l}\text { Barridos genómicos en familias: estudios de } \\
\text { ligamiento (enfoque no-paramétrico) } \\
\text { (cita 21) }\end{array}$ & $\begin{array}{l}\text { Meta-análisis } \\
\quad \text { Cita } 30\end{array}$ \\
\hline $\begin{array}{l}\text { Barridos genómicos en poblaciones aisladas } \\
\text { (cita 18) }\end{array}$ & $\begin{array}{l}\text { Genotipado intenso de genes candidatos } \\
\text { ("Gene Centric" Human CVD BeadChip) } \\
\text { Cita } 31\end{array}$ \\
\hline $\begin{array}{l}\text { Barridos genómicos en estudios de asociación } \\
\text { en poblaciones abiertas } \\
\text { (citas 26-29) }\end{array}$ & $\begin{array}{l}\text { Randomización mendeliana } \\
\text { (cita 8) }\end{array}$ \\
\hline
\end{tabular}

SNP: Single Nucleotide Polymorphisms; CNV: Copy-Number Variation.

El artículo de Saavedra y cols $^{35}$, publicado en este número de la Revista Chilena de Cardiología se centra en los polimorfismos del gen $A P O A 5^{35}$. Tal como señalan los autores de este artículo, existen evidencias sólidas provenientes de estudios de diferente naturaleza que relacionan a la apolipoproteína $A-V$ y a la variabilidad genética de APOA5 en la regulación de los niveles de TG plasmáticos, por lo que las variantes de este gen se han erigido como un referente en los cálculos del poder predictivo de la variabilidad genética sobre la trigliceridemia1. Por un lado, se han descrito mutaciones raras en APOA5 con un efecto de penetrancia variable sobre la hipertrigliceridemia severa $^{36}$, mientras que adicionalmente, también se ha descrito un efecto relativamente consistente de las variantes $-1131 \mathrm{~T}>\mathrm{C}$ y $\mathrm{S} 19 \mathrm{~W}$ sobre los niveles plasmáticos de $\mathrm{TG}^{1}$. El artículo de Saavedra y cols, apunta en este mismo sentido al informar la asociación entre estas variantes genéticas con los TG plasmáticos, representando una muy importante contribución al conocimiento del efecto de estas variantes genéticas y su distribución en la población Chilena. Es importante destacar la cuidada caracterización fenotípica y genotípica aplicada en este estudio y el importante esfuerzo de selección de participantes que ha conducido a un tamaño de muestra considerable. A pesar de esto y como suele ser común en estudios de epidemiología genética en la literatura internacional, es difícil poder alcanzar tamaños de muestra adecuados que permitan tener un poder estadístico suficiente para estimar con precisión la magnitud de los efectos principales, así como permitir el control de variables confundentes y la evaluación de las posibles interacciones entre las variables participantes.

Igualmente, es importante resaltar la importancia de la subestructura genética poblacional en estudios de epidemiología genética en nuestra población ${ }^{37}$, tanto por el posible efecto de confusión de la estratificación genética poblacional, como por la posibilidad de existencia de variantes genéticas relevantes en grupos étnicos específicos, como 
aparentemente ocurre con la asociación entre la variante $185 \mathrm{G}>\mathrm{C}$ de APOA5 con los niveles de TG en poblaciones asiáticas ${ }^{38}$. Otro hallazgo relevante del estudio de Saavedra y cols, es la asociación encontrada entre los genotipos $-1131 \mathrm{~T}>\mathrm{C}$ y $\mathrm{S} 19 \mathrm{~W}$ de APOA5 y los niveles de glucosa plasmática en ayunas. En este sentido, se ha descrito una relación entre la acción de LPL y la dislipidemia diabética y la resistencia a la insulina ${ }^{15,39}$. Por otro lado, es interesante también señalar que algunas de las señales significativas procedentes de los estudios de asociación de genoma completo en relación a los niveles de TG plasmáticos se refieren a genes relacionados con el metabolismo de los hidratos de carbono tales como GCKR y MLXIPL ${ }^{26-29}$. El gen GCKR codifica para la proteína reguladora de la glucoquinasa, que es la enzima que actúa como sensor de la glucosa plasmática y que participa en la fosforilación de la glucosa en la primera etapa de la glicolisis, mientras que el gen MLXIPL codifica para un factor de transcripción sensible a la acción de la glucosa.

\section{REFERENCIAS:}

1. HEGELE. Plasma lipoproteins: genetic influences and clinical implications. Nat Rev Genet 2009; 10: 109-121.

2. SUNG J, LEE K, SONG YM. Heritabilities of the metabolic syndrome phenotypes and related factors in Korean twins. J Clin Endocrinol Metab 2009; 94: 4946-4952. Nutr Metab Cardiovasc Dis. 2009 Sep;19(7): 455-61. Epub 2009 Feb 6.

3. FENGER M, BENYAMIN B, SCHOUSBOE K, SORENSEN TI, KYVIK KO. Variance decomposition of apolipoproteins and lipids in Danish twins. Atherosclerosis 2007; 191: 40-47.

4. SOUREN NY, ZEEGERS MP, JANSSEN RG, STEYLS A, GIELEN M, LOOS RJ, et al. Anthropometry, carbohydrate and lipid metabolism in the East Flanders Prospective Twin Survey: linkage of candidate genes using two sibpair based variance components analyses. Twin Res Hum Genet 2008; 11: 505-516.

5. VAN WIJK DF, STROES ES, KASTELEIN JJ. Lipid measures and cardiovascular disease prediction. Dis Markers 2009; 26: 209-216.

6. CALABRESI L, BALDASSARRE D, CASTELNUOVO $S$. Functional lecithin: cholesterol acyltransferase is not required for efficient atheroprotection in humans. Circulation 2009; 120: 628-635.

7. WEISGRABER KH, RALL SC JR, BERSOT TP, MAHLEY RW, FRANCESCHINI G, SIRTORI CR, et al. Apolipoprotein A-IMilano. Detection of normal A-I in affected subjects and evidence for a cysteine for arginine substitution in the variant A-I. J Biol Chem 1983; 258: 2508-2513.

8. FRIKKE-SCHMIDTR. Genetic variation in the ABCA1 gene, HDL cholesterol, and risk of ischemic heart disease in the general population. Atherosclerosis 2009 (epub ahead of print).

9. SARWARN, DANESHJ, EIRIKSDOTTIR G, SIGURDSSON G, WAREHAM N, BINGHAM S, et al. Triglycerides and the risk of coronary heart disease. 10158 incident cases among 262525 participants in 29 Western prospective studies. Circulation 2007; 115: 450-458.
10. BANSAL S, BURING JE, RIFAI N, MORA S, SACKS FM, RIDKER PM. Fasting compared with nonfasting triglycerides and risk of cardiovascular events in women. JAMA 2007; 298:309-316.

11. PÉREZ-MARTINEZ P, LOPEZ-MIRANDA J, PÉREZJIMÉNEZ F, ORDOVAS JM. Influence of genetic factors in the modulation of postprandial lipemia. Atheroscler Suppl 2008; 9: 49-55.

12. ARTEAGA A, CUEVAS A, RIGOTTI A, GONZÁLEZ F, CASTILLO S, MATA P, ALONSO R. Hipercolesterolemia familiar heterocigota: diagnóstico molecular y terapia hipolipemiante combinada. Caso clínico. Rev Med Chile 2007; 135: 216-220.

13. RAHALKAR AR, GIFFEN F, HAR B, HO J, MORRISON KM, HILL J, et al. Novel LPL mutations associated with lipoprotein lipase deficiency: two case reports and a literature review. Can J Physiol Pharmacol 2009; 87 : 151-160.

14. XIE C, WANG ZC, LIU XF, YANG MS. The common biological basis for common complex diseases: evidence from lipoprotein lipase gene. Eur J Hum Genet 2010; 18: 3-7.

15. BRECKENRIDGE WC, LITTLE JA, STEINER G, CHOW A, POAPST M. Hypertriglyceridemia associated with deficiency of apolipoprotein C-II. N Engl J Med 1978; 298: 1265-1273.

16. WALDEN CC, HEGELE RA. Apolipoprotein $E$ in hyperlipidemia. Ann Intern Med 1994; 120: 1026-36.

17. JONG MC, HOFKER MH, HAVEKES LM. Role of ApoCs in lipoprotein metabolism: differences between ApoC1, ApoC2 and ApoC3. Atheroscler Thromb Vasc Biol 1999; 19: 472-484.

18. POLLIN TI, DAMCOTT CM, SHEN H, OTT SH, SHELTON J, HORENSTEIN RB, ET AL. A null mutation in human APOC3 confers a favorable plasma lipid profile and apparent cardioprotection. Science 2008; 322: 1702-1705.

19. NEWMAN DL, ABNEY M, DYTCH H, RODNEY P, McPEAK MS, OBER C. Major loci influencing serum triglyceride 
levels on $2 q 14$ and 9p21 localized by homozygosityby-descent mapping in a large Hutterite pedigree. Hum Mol Genet 2003; 12: 137-144.

20. NG SB, BUCKINGHAM KJ, LEE C, BIGHAM AW, TABOR HK, DENT KM, et al. Exome sequencing identifies the cause of a mendelian disorder. Nat Genet 2010; 42: 30-35.

21. COLETTA DK, SCHNEIDER J, HU SL, DYER TD, PUPPALA S, FAROOK VS, et al. Genome-wide linkage scan for genes influencing plasma triglyceride levels in the veterans administration genetic epidemiology study. Diabetes 2009; 58: 279-284

22. FRAZER KA, MURRAY SS, SCHORK NJ, TOPOL EJ. Human genetic variation and its contribution to complex traits. Nat Genet Rev 2009; 10: 241-251.

23. BOTSTEIN D, RISCH N. Discovering genotypes underlying human phenotypes: past successes for mendelian disease, future approaches for complex disease. Nat Genet 2003; 33: 228-37.

24. REDDEN DT, ALLISON DB. Nonreplication in genetic association studies of obesity and diabetes research. J Nutr 2003; 133: 3323-3326.

25. THE WELLCOME TRUST CASE CONTROL CONSORTIUM. Genome-wide association study of 14,000 cases of seven common diseases and 3,000 shared controls. Nature 2007: 447: 661-678.

26. KOONER JS, CHAMBERS JC, AGUILAR-SALINAS CA, HINDS DA, HYDE CL, WARNES GR, et al. Genome-wide scan identifies variation in MLXIPL associated with plasma triglycerides. Nat Genet 2008; 40: 149-151.

27. WALLACE C, NEWHOUSE SJ, BRAUND P, ZHANG F, TOBIN M, FALCHI M, et al. Genome-wide Association Study Identifies Genes for Biomarkers of Cardiovascular Disease: Serum Urate and Dyslipidemia. Am J Hum Genet 2008; 82: 139-149.

28. AULCHENKO YS, RIPATTI S, LINDQUIST I, BOOMSMA D, HEID IM, PRAMSTALLER PP, et al. Loci influencing lipid levels and coronary heart disease risk in 16 European population cohorts. Nat Genet 2009; 41: 47-55.

29. KATHIRESAN S, WILLER CJ, PELOSO G, DEMISSIE S, MUSUNURU K, SCHADT E, et al. Common variants at 30 loci contribute to polygenic dyslipidemia. Nat Genet 2009; 41: 56-65.

30. BENNET AM, DI ANGELANTONIO E, YE Z, WENSLEY F,
DAHLIN A, AHLBOM A, et al. Association of apolipoprotein E genotypes with lipid levels and coronary risk. JAMA 2007; 298: 1300-1311.

31. TALMUD PJ, DRENOS F, SHAH S, SHAH T, PALMEN J, VERZILLI C, et al. Gene-centric association signals for lipids and apolipoproteins identified via the HumanCVD Beadchip. Am J Hum Genet 2009; 85: 628-642.

32. BECKMANN JS, ESTIVILL X, ANTONARAKIS SE. Copy number variants and genetic traits: closer to the resolution of phenotypic to genotypic variability. Nat Rev Genet 2007; 8: 639-646.

33. LANKTREE MB, RAJAKUMAR C, BRUNT JH, KOSCHINSKY ML, CONNELLY PW, HEGELA RA. Determination of lipoprotein(a) kringle repeat number from genomic DNA: copy number variation genotyping using qPCR. J Lipid Res 2009; 50: 768-772.

34. CLARKE R, PEDEN JF, HOPEWELL JC, KYRIAKOU T, GOEL A, HEATH SC, et al. Genetic variants associated with $\mathrm{Lp}(\mathrm{a})$ lipoprotein level and coronary disease. N Engl J Med 2009; 361: 2518-2528

35. SAAVEDRA C, HERNANDEZA, CAAMAÑO J, JARAMILLO $P$, LANAS F, SALAZAR LA. Polimorfismos genéticos de APOA5 se asocian a hipertrigliceridemia e hiperglicemia en individuos chilenos con enfermedad coronaria y controles. Rev Chil Cardiol 2010; 29: 19-27

36. TALMUD PJ. Rare APOA5 mutations--clinical consequences, metabolic and functional effects: an ENID review. Atherosclerosis 2007; 194: 287-292.

37. SANTOS JL, PÉREZ-BRAVO F, CARRASCO E, CALVILLÁN M, ALBALA C. Association between type 1 diabetes and HLA-DQB1 alleles in a case-parents study conducted in Santiago, Chile. Am J Epidemiol 2001; 153: 794-798.

38. PULLINGER CR, AOUIZERAT BE, MOVSESYAN I, DURLACH V, SIJBRANDS EJ, NAKAJIMA K, et al. An apolipoprotein A-V gene SNP is associated with marked hypertriglyceridemia among Asian-American patients. J Lipid Res 2008; 49:1846-1854.

39. MCLEAN AG, PETERSONS CJ, HOOPER AJ, BURNETT JR, BURT MG, DOOGUE MP. Extreme diabetic lipaemia associated with a novel lipoprotein lipase gene mutation. Clin Chim Acta 2009; 406: 167-169. 